\title{
Synchronization Transitions in a Disordered Josephson Series Array
}

\author{
Kurt Wiesenfeld \\ School of Physics, Georgia Institute of Technology, Atlanta, Georgia 30332 \\ Pere Colet \\ Instituto de Estructura de la Materia, Consejo Superior de Investigaciones Científicas, Serrano 123, E-28006 Madrid, Spain
}

Steven H. Strogatz

Theoretical and Applied Mechanics, Kimball Hall, Cornell University, Ithaca, New York 14853

(Received 28 July 1995)

\begin{abstract}
We show that a current-biased series array of nonidentical Josephson junctions undergoes two transitions as a function of the spread of natural frequencies. One transition corresponds to the onset of partial synchronization, and the other corresponds to complete phase locking. In the limit of weak coupling and disorder, the system can be mapped onto an exactly solvable model introduced by Kuramoto and the transition points can be accurately predicted.
\end{abstract}

PACS numbers: 05.45.+b, 74.40.+k, 74.50.+r

Populations of coupled nonlinear oscillators can spontaneously synchronize to a common frequency, despite differences in their natural frequencies. This remarkable phenomenon, known as collective synchronization, has been observed in many physical and biological systems, including relaxation oscillator circuits, networks of neurons and cardiac pacemaker cells, chorusing crickets, and fireflies that flash in unison [1,2].

In a pioneering study, Winfree [3] developed a mathematical framework for studying large populations of limitcycle oscillators, and he showed that the onset of synchronization is analogous to a thermodynamic phase transition. This observation was refined by Kuramoto [4] who proposed and analyzed an exactly solvable mean-field model of coupled oscillators with distributed natural frequencies. The Kuramoto model has stimulated a great deal of theoretical work [5-12], thanks to its analytical tractability, but it has not been used to describe any experimental system.

In this Letter we show that a series array of Josephson junctions provides a physical realization of the Kuramoto model. This connection allows us to give the first analytical treatment of mutual synchronization in a Josephson array for the realistic case where the junctions are nonidentical. We find that the array displays two transitions: the first corresponds to the onset of dynamical order, while the second coincides with total phase locking and the quenching of fluctuations. We calculate that both of these transitions are experimentally accessible with existing technology.

Consider a series array of $N$ junctions, biased with a constant current $I_{B}$ and subject to a load with inductance $L$, resistance $R$, and capacitance $C$. For resistively shunted junctions with negligible capacitance, the governing circuit equations are

$$
\frac{\hbar}{2 e r_{j}} \dot{\phi}_{j}+I_{j} \sin \phi_{j}+\dot{Q}=I_{B}, \quad j=1, \ldots, N,
$$

$$
L \ddot{Q}+R \dot{Q}+\frac{1}{C} Q=\frac{\hbar}{2 e} \sum_{k=1}^{N} \dot{\phi}_{k},
$$

where $\phi_{j}$ is the wave-function phase difference across the $j$ th Josephson junction, $r_{j}$ is the junction resistance, $I_{j}$ is the junction critical current, $Q$ is the charge on the load capacitor, $\hbar$ is Planck's constant divided by $2 \pi$, and $e$ is the elementary charge. The overdot denotes differentiation with respect to time. The voltage drop across the $j$ th junction is $(\hbar / 2 e) \dot{\phi}_{j}$. Note that the allto-all coupling in Eq. (2) arises naturally from the circuit analysis rather than from a mean-field approximation.

The key feature of the dynamics is the competition between the intrinsic disorder (i.e., variations in the junction resistances and critical currents) and the coupling between the junctions mediated by the load. In the absence of a load the junctions are dynamically uncoupled and (for $I_{B}>I_{j}$ ) the $j$ th element executes voltage oscillations at its bare frequency $\omega_{j}=\left(2 e r_{j} / \hbar\right)\left(I_{B}^{2}-I_{j}^{2}\right)^{1 / 2}$. With the load, however, the nonlinear interactions cause the elements to oscillate at shifted (dressed) frequencies $\left\{\tilde{\omega}_{j}\right\}$. In particular, junctions with different bare frequencies can oscillate at a common frequency. Typically this requires the coupling to be large enough to overcome the intrinsic spread in the natural frequencies $\left\{\omega_{j}\right\}$; the larger the coupling, the greater the number of elements entrained to a common frequency. In the design of useful highfrequency voltage sources the goal is to achieve complete frequency locking (and in addition, stable in-phase oscillations).

Figure 1 shows the results of simulations using typical parameter values for Josephson junctions. Shown is the fraction of junctions locked to a single common frequency as a function of the spread $\Delta$ in critical currents. (Junctions $i$ and $j$ are frequency locked if $\phi_{j}-\phi_{i}$ remains bounded as $t \rightarrow \infty$.) The critical currents were chosen to match a normalized parabolic distribution with 


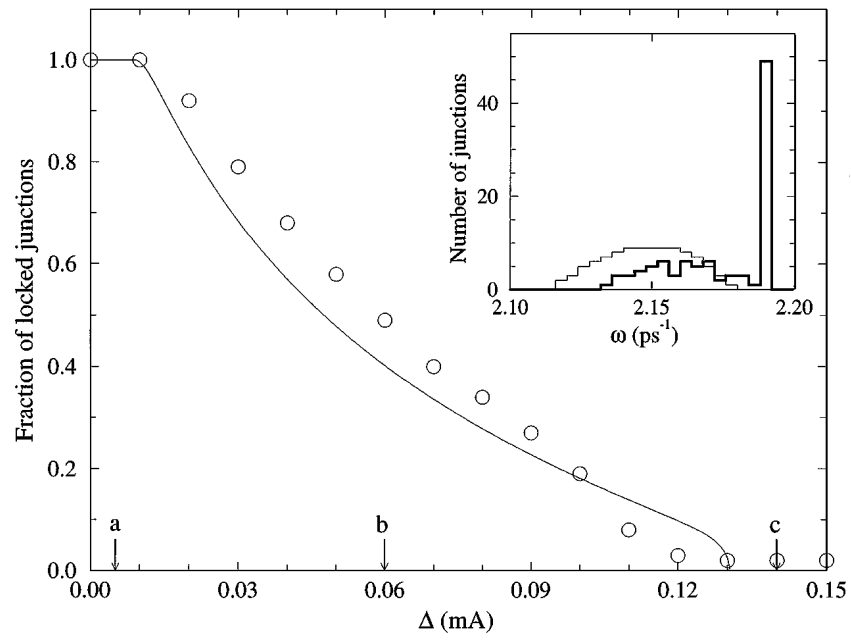

FIG. 1. Fraction of junctions locked to a common frequency as a function of the spread $\Delta$ of critical currents (see text); $N=$ $100, I_{B}=1.5 \mathrm{~mA}, R=50 \Omega, L=25 \mathrm{pH}, C=0.04 \mathrm{pF}, \bar{I}=$ $0.5 \mathrm{~mA}$, and for all junctions, $r_{i}=0.5 \Omega$. Circles correspond to numerical simulations of Eqs. (1) and (2). The solid line corresponds to Eq. (12) (see text). Power spectra for regimes (a), (b), and (c) are shown in Fig. 2. The inset shows histograms for the bare (thin line) and dressed (thick line) frequencies at the point $\Delta=0.06 \mathrm{~mA}$.

mean $\bar{I}$ and full width $2 \Delta$

$$
P(I)=\frac{3}{4 \Delta^{3}}\left[\Delta^{2}-(I-\bar{I})^{2}\right] .
$$

There are three different dynamical regimes. Decreasing the disorder from a large value there is a transition at $\Delta=\Delta_{c}$ signaling the onset of frequency locking; for $\Delta_{L}<\Delta<\Delta_{c}$ there is partial frequency locking; for $\Delta<\Delta_{L}$ the frequency locking is complete. The inset shows the distribution of bare and dressed frequencies at $\Delta=0.06$ where about half of the junctions are locked.

Figure 2 shows the corresponding power spectra for the total voltage across the array, for representative points in the three regimes [labeled (a), (b), and (c) in Fig. 1]. One sees that the onset of order is signaled by the birth of a sharp spike at the locking frequency [compare Figs. 2(b) and 2(c)], while the complete-locking transition coincides with the quenching of low-frequency fluctuations [compare Figs. 2(a) and 2(b)]. We have also run these simulations including Johnson noise on $r_{j}$ and $R$ for a temperature of $4 \mathrm{~K}$ : Fig. 2 simply acquires a flat noise floor at $10^{-5}$.

We now show that the Josephson system can be mapped onto Kuramoto's model, a connection which enables us to make quantitative predictions about the observed dynamics. This can be done in the limit of weak coupling and disorder by extending an averaging procedure previously applied to identical junction arrays. The first step is to introduce "natural angles" $\theta_{j}$ defined by

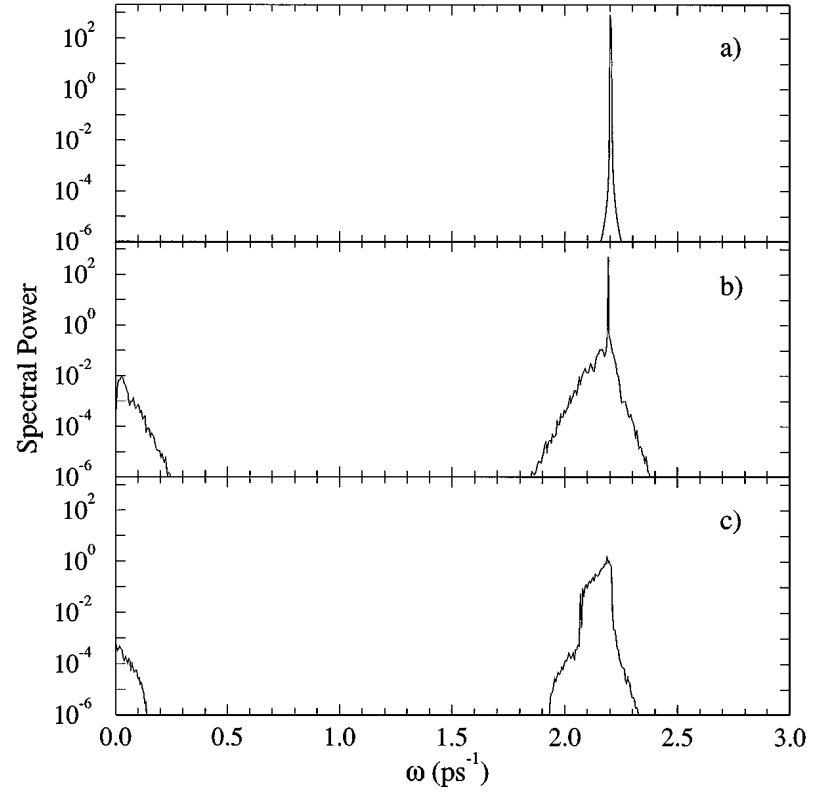

FIG. 2. Power spectra for the ac component of the total array voltage, $(\hbar / 2 e)\left(\sum \dot{\phi}_{k}-\left\langle\sum \dot{\phi}_{k}\right\rangle\right)$, where $\langle\cdots\rangle$ indicates time average, for the three different regimes of Fig. 1 . (a) $\Delta=$ $0.005 \mathrm{~mA}$. (b) $\Delta=0.06 \mathrm{~mA}$. (c) $\Delta=0.14 \mathrm{~mA}$.

$$
\frac{d \theta_{j}}{\omega_{j}}=\frac{d \phi_{j}}{\left(2 e r_{j} / \hbar\right)\left(I_{B}-I_{j} \sin \phi_{j}\right)} .
$$

The angles $\theta_{j}$ are natural in the sense that (in the uncoupled limit) they undergo uniform rotation, while the $\phi_{j}$ do not. Using the trigonometric relation $I_{B}-$ $I_{j} \sin \phi_{j}=\left(I_{B}^{2}-I_{j}^{2}\right) /\left(I_{B}-I_{j} \cos \theta_{j}\right)$ obtained by integrating Eq. (4), Eq. (1) becomes

$$
\dot{\theta}_{j}=\omega_{j}-\frac{\omega_{j} \dot{Q}}{I_{B}^{2}-I_{j}^{2}}\left(I_{B}-I_{j} \cos \theta_{j}\right) .
$$

In the weak-coupling limit, and for identical junctions $\left(I_{j}=I, r_{j}=r, \omega_{j}=\omega\right)$, Swift and co-workers have shown how this system can be reduced using the method of averaging [13,14]. The basic idea is that one can replace the coupling terms on the right side by their time average over one period, which generates higher order corrections. The variable $Q$, meanwhile, can be eliminated by noting that the load equation (2) is just a quasiperiodically driven linear oscillator, which can be solved explicitly using the uncoupled solution of Eq. (5). As shown in [14], the first-order averaged version of Eq. (5) is

$$
\dot{\theta}_{j}=\omega-\frac{K}{N} \sum_{k=1}^{N} \sin \left(\theta_{j}-\theta_{k}+\alpha\right),
$$

where

$$
K=\frac{N r \omega\left(2 e r I_{B} / \hbar-\omega\right)}{\left[\left(L \omega^{2}-1 / C\right)^{2}+\omega^{2}(R+N r)^{2}\right]^{1 / 2}}
$$


and

$$
\cos \alpha=\frac{L \omega^{2}-1 / C}{\left[\left(L \omega^{2}-1 / C\right)^{2}+\omega^{2}(R+N r)^{2}\right]^{1 / 2}},
$$

where $-\pi / 2 \leq \alpha \leq 0$. The same steps can be carried out for the case of small disorder, with the result

$$
\dot{\theta}_{j}=\omega_{j}-\frac{K}{N} \sum_{k=1}^{N} \sin \left(\theta_{j}-\theta_{k}+\alpha\right),
$$

where $K$ and $\cos \alpha$ are given by the same expressions (7) and (8) provided the quantities $I, r$, and $\omega$ are replaced, respectively, by the mean values of $I_{j}, r_{j}$, and $\omega_{j}(\bar{I}$, $\bar{r}_{j}$, and $\left.\bar{\omega}\right)$. In the small disorder regime that we are considering, $\bar{\omega}=(2 e \bar{r} / \hbar)\left(I_{B}^{2}-\bar{I}^{2}\right)^{1 / 2}$.

Equation (9) is the Kuramoto model [15]. A great deal is known about the dynamics of this system in the large- $N$ limit. A useful measure of the coherence of the array is the complex order parameter $\sigma e^{i \psi}=(1 / N) \sum_{j} e^{i \theta_{j}}$. For a unimodal bare frequency distribution $g(\omega)$, and in the large- $N$ limit, $\sigma$ settles down to a constant value and $\psi$ rotates uniformly, $\psi=\Omega t$. Sakaguchi and Kuramoto [6] showed that the quantities $\sigma$ and $\Omega$ can be determined from the self-consistency relation

$\sigma e^{i \alpha}=K \sigma\left[i J+\int_{-\pi / 2}^{\pi / 2} d \xi g(\Omega+K \sigma \sin \xi) e^{i \xi} \cos \xi\right]$,

where

$$
J=\int_{0}^{\pi / 2} d \xi \frac{\cos \xi(1-\cos \xi)}{\sin ^{3} \xi}[g(\Omega+\mu)-g(\Omega-\mu)],
$$

with $\mu=K \sigma / \sin \xi$. Specifically, $\sigma$ acts as an order parameter for the onset of frequency locking; the critical point $K=K_{c}$ is where the $\sigma>0$ solution of Eq. (10) branches off of the $\sigma=0$ solution. The locking frequency $\Omega$ can be shifted from the mean bare frequency $\bar{\omega}$, as seen in Fig. 1 (inset). In the Kuramoto model this effect is due to the nonzero value for $\alpha$, as can be seen from the simple case without disorder: from Eq. (9), the in-phase solution is $\theta_{j}=(\bar{\omega}-K \sin \alpha) t$, so that the locking frequency is given by $\Omega=\bar{\omega}-K \sin \alpha$.

Meanwhile, the fraction $f$ of locked oscillators is simply

$$
f=\int_{\Omega-K \sigma}^{\Omega+K \sigma} d \omega g(\omega)
$$

so that the transition point $K_{L}$ is the smallest $K$ for which $f=1$. In the special case where $\alpha=0$ and $g(\omega)$ is symmetric, further analytic progress is possible (since then $\Omega$ and $J=0$ ), but more typically Eqs. (10) and (12) need to be solved numerically to determine the critical points. Note that $K_{c}$ depends on the local properties of $g(\omega)$ near its mean, while $K_{L}$ is sensitive to the outlying values of $\omega_{j}$ : in fact, in the $N \rightarrow \infty$ limit this transition exists only if $g(\omega)$ has finite support.
Having established the connection with the Kuramoto model, we are in a position to make quantitative predictions about the dynamical transitions in the Josephson array. For the parameter values used to generate Fig. 1, the corresponding Kuramoto parameters are $K=0.0601$ and $\cos \alpha=0.3878$. The solid line of Fig. 1 shows the prediction from Eqs. (10) and (12), for the fraction of locked junctions which agrees fairly well with the simulations.

We turn next to the possibility of experimental observation of these transitions in Josephson arrays. Again the connection with the Kuramoto model gives us substantial insight. The first consideration is that in the in-phase state should be an attractor when there is no disorder $\left(\omega_{j}=\bar{\omega}\right)$. A linear stability analysis shows that this is stable if and only if $K \cos \alpha>0$. From Eq. (8) this requires that $1 / \sqrt{L C}<\bar{\omega}$. Second, in order to optimize the sensitivity of the measurements, we want to maximize the power delivered to the load, which entails matching the load and array resistances, so $R=N \bar{r}$. Third, for superconductor-normal-superconductor junctions, fluctuations in the critical currents $I_{j}$ are linked to those in the resistances $r_{j}$, such that the product $I_{j} r_{j}$ is fixed for all the junctions [16]. For a given $\Delta$ this increases the spread in $\left\{\omega_{j}\right\}$ and so increases the effective disorder. Fourth, it is not practical to vary the level of disorder; rather, the most natural control parameter is the bias current $I_{B}$. Of course, varying $I_{B}$ affects simultaneously the coupling strength $K$, the phase shift $\alpha$, and the bare frequencies $\omega_{j}$. These can be determined according to the explicit formulas given above. Generally speaking, as $I_{B}$ decreases from a large value, the trend is that the frequency spread decreases, whereas the effective coupling strength $K \cos \alpha$ first increases, goes through a maximum, and then drops to zero at the point where the in-phase state loses stability, at $\omega=(L C)^{-1 / 2}$.

Fifth, it is not practical to measure directly the number of frequency-locked junctions; however, the transitions should be observable by measuring the frequency spectrum of the total voltage across the load. Specifically, the onset of order is signaled by the birth of a narrow line at frequency $\Omega$ [compare Figs. 2(b) and 2(c)]. The strength $A_{\Omega}$ of this line is proportional to the Kuramoto order parameter $\sigma$ :

$$
A_{\Omega}=2 K \sigma \frac{I_{B}^{2}-\bar{I}^{2}}{\bar{\omega}^{2} \bar{I}} \sqrt{\left(L \Omega^{2}-1 / C\right)^{2}+\Omega^{2} R^{2}} .
$$

Thus, $A_{\Omega}$ is a good order parameter for determining the onset of coherence. On the other hand, $A_{\Omega}$ shows no dramatic change at the complete-locking transition, and it is better to monitor the broadband low-frequency part of the voltage output, which is quenched at this transition [compare Figs. 2(a) and 2(b)].

Figure 3 shows the results of simulations that take into account all of the above considerations. Figure 3(a) plots $A_{\Omega}$ and, for comparison, Fig. 3(b) plots the fraction of locked junctions. The junction critical currents were 


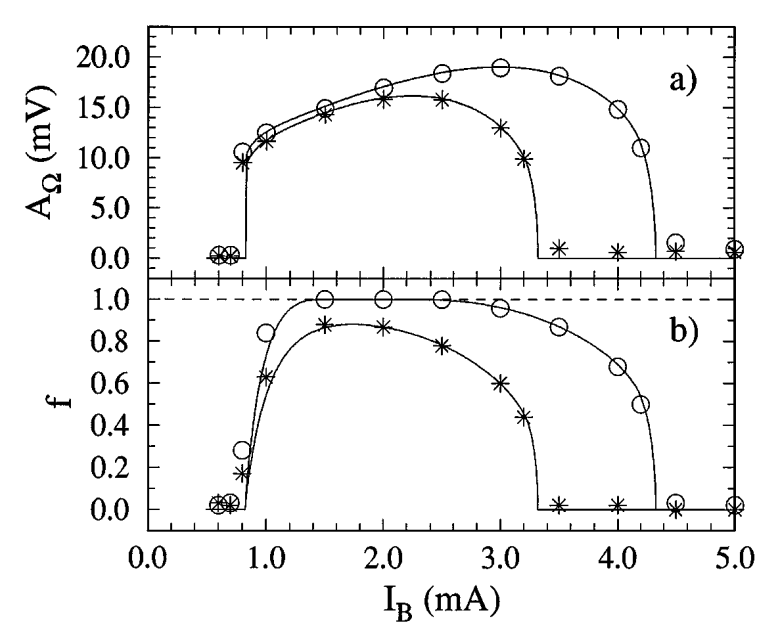

FIG. 3. Dependence of (a) $A_{\Omega}$ and (b) fraction of locked junctions vs the bias current $I_{B}$, for $N=100, R=50 \Omega$, $L=25 \mathrm{pH}, C=0.04 \mathrm{pF}, \bar{I}=0.5 \mathrm{~mA}$, and $\bar{r}=0.5 \Omega$. The solid lines correspond to Eq. (13) in (a) and to Eq. (12) in (b). Symbols correspond to numerical simulations of Eqs. (1) and (2), circles for $\Delta=0.001 \mathrm{~mA}$, and asterisks for $\Delta=$ $0.002 \mathrm{~mA}$.

chosen as before, according to Eq. (3), and the resistances were taken such that $r_{j}=\bar{r} \bar{I} / I_{j}$. There are two regimes where $A_{\Omega}$ is very small. For $\Delta=0.001 \mathrm{~mA}$ (circles), the transitions marking the onset of order (at $I_{B} \approx 4.3 \mathrm{~mA}$ ) and the loss of dynamical stability (at $I_{B} \approx 0.8 \mathrm{~mA}$, still well above the critical current $0.5 \mathrm{~mA}$ ) are clearly visible in the behavior of $A_{\Omega}$. The transitions into and out of the fully locked state, as expected, are not reflected in $A_{\Omega}$. For $\Delta=0.002 \mathrm{~mA}$ (asterisks) full locking never takes place whereas the other transitions are still clearly visible [17]. For both data sets the theory does an excellent job. The power delivered to the load at the locking frequency $\Omega$ is $P_{\Omega}=f A_{\Omega}^{2} / 2 R$; for these parameters this is about $30 \mathrm{nW}$ per junction. This should be sufficient power to detect using on-chip measurements.

Recently, Watanabe and Swift [18] and Chernikov and Schmidt [19] showed how to extend the averaging method (for arrays of identical junctions) to include junction capacitance. For sufficiently large bias currents, they again recover an equation of the form (6). Consequently, in the presence of weak disorder, the system can again be related to the Kuramoto model, and the dynamical transitions described above are expected. Also of great current interest is the effect of disorder on sychronization in two-dimensional arrays [20,21]; it is an open question whether those systems can be related to the Kuramoto model.

We thank Sam Benz for numerous helpful discussions on the physics of Josephson junction arrays. Research supported in part by ONR under Contract No. N00014J-1257, NATO Collaborative Research Grant No. CRG950282, and by NSF Grants No. DMS-9057433 and No. DMS-9500948.

[1] A.T. Winfree, The Geometry of Biological Time (Springer, New York, 1980).

[2] S. H. Strogatz and I. Stewart, Sci. Am. 269, No. 6, 102 (1993).

[3] A. T. Winfree, J. Theor. Biol. 16, 15 (1967).

[4] Y. Kuramoto, in Proceedings of the International Symposium on Mathematical Problems in Theoretical Physics, edited by H. Araki, Lecture Notes in Physics Vol. 39 (Springer, Berlin, 1975); Chemical Oscillations, Waves, and Turbulence (Springer, Berlin, 1984).

[5] For a review, see S. H. Strogatz, in Frontiers in Mathematical Biology, edited by S. Levin, Lecture Notes in Biomathematics Vol. 100 (Springer, Berlin, 1994).

[6] H. Sakaguchi and Y. Kuramoto, Prog. Theor. Phys. 76, 576 (1986).

[7] S. H. Strogatz, R. E. Mirollo, and P.C. Matthews, Phys. Rev. Lett. 68, 2730 (1992).

[8] S. H. Strogatz and R.E. Mirollo, J. Stat. Phys. 63, 613 (1991).

[9] L. L. Bonilla, J. C. Neu, and R. Spigler, J. Stat. Phys. 67, 313 (1992).

[10] J. L. van Hemmen and W. F. Wreszinski, J. Stat. Phys. 72, 145 (1993).

[11] J. D. Crawford, J. Stat. Phys. 74, 1047 (1994); Phys. Rev. Lett. 74, 4341 (1995).

[12] H. Daido, Prog. Theor. Phys. 88, 1213 (1992); Phys. Rev. Lett. 73, 760 (1994).

[13] J. W. Swift, S. H. Strogatz, and K. Wiesenfeld, Physica (Amsterdam) 55D, 239 (1992).

[14] K. Wiesenfeld and J. W. Swift, Phys. Rev. E 51, 1020 (1995).

[15] To be more precise, the original Kuramoto model [4] assumed $\alpha=0$; the generalization to $\alpha \neq 0$ was studied by Sakaguchi and Kuramoto [6].

[16] S. P. Benz, "Superconductor Normal Superconductor Junction for Programmable Voltage Standards" (to be published).

[17] For larger critical currents (e.g., $2 \mathrm{~mA}$ ) full locking occurs at larger values of $\Delta / \bar{I}$ (e.g., $2 \%$ ). This is in the range of present fabrication techniques; see P. A. A. Booi and S. P. Benz, Appl. Phys. Lett. 64, 2163 (1994).

[18] S. Watanabe and J.W. Swift, J. Nonlin. Sci. (to be published).

[19] A. A. Chernikov and G. Schmidt, Phys. Rev. E 52, 3415 (1995).

[20] M. Octavio, C. B. Whan, and C. J. Lobb, Appl. Phys. Lett. 60, 766 (1992).

[21] R. L. Kautz, IEEE Trans. Appl. Supercon. 5, 2702 (1995). 